same topics covered by Sim are dealt with, usually at greater length; in addition an account of membrane functions is included. It is disappointing, however, that the development of the dynamic picture of the membrane organization seldom leads to a full description of a membrane function. This is particularly noticeable in the chapter on transport processes, although there are good introductions to some of the more physiological functions. The omission of a full reference list is serious and there are also too many contradictions, repetition and other careless errors. Despite its faults Dynamics of Biological Membranes should be provocative reading for those, especially postgraduate students, doing research on membranes but the lack of references is a real nuisance for teachers, and the errors and variability in treatment are a drawback for undergraduates.

Chloroplasts and Mitochondria by Tribe and Whittaker is intended to introduce sixth-form students to the structure and assembly of these organelles as well as to their roles in photosynthesis and respiration. The book starts with a confusing and laboured account of the role of ATP in bioenergetics which is followed by errors and poor judgement in the chapter on chemical reactions, including an inaccurate description of chemiosmotic coupling. Several of the photographs are far from clear, the separate chapter on history is of doubtful worth and the final chapter on practical work is too brief to give great hope of success. In all, the book is very far from ideal for its intended readers who are too inexperienced to cope with the deficiencies.

In Mitochondria, Chloroplasts and Bacterial Membranes, Prebble recognizes the similarities between the two organelles and bacteria and the value of a combined treatment. He gives comprehensive and accurate descriptions of the metabolic, bioenergetic, transport and biosynthetic processes of mitochondria and chloroplasts but has had to be highly selective in dealing with bacteria. Two relatively short chapters deal with bacterial energy transformation and bacterial photosynthesis. Two pages on bacterial transport processes seems excessively short and I thought it rather mean to include mitochondrial evolution in a bacterial chapter - a final chapter on the evolution of mitochondria and chloroplasts would have been a great improvement.

Overall this is a valuable compendium of information on chloroplasts and mitochondria combined with an introduction to bacterial bioenergetics. It should be useful to university teachers, students and research workers, but more so for those working on mitochondria or chloroplasts than for those whose interests lie with the bacteria.

Michael Selwyn is Dean of the School of Biological Sciences at the University of East Anglia.

\section{Neuro this, that and the other}

\section{Maria Fitzgerald}

Principles of Neuroanatomy.

By Jay B. Angevine, Jr. and Carl W. Cotman.

Oxford University Press: 1982. Pp.393. Hbk \$32.50; pbk

$\$ 17.95, £ 8.95$.

Basic Neurochemistry, 3rd Edn.

Edited by George J. Siegel et al.

Little, Brown: 1982. Pp.826. \$30.75.

An Introduction to Neurophysiology.

By J.F. Stein.

Blackwell Scientific/Mosby: 1982.

Pp.385. f12, \$19.95.

Comparative Neurobiology.

By P.J. Mill.

Edward Arnold: 1982. Pp.280. £9.95, \$19.95.

THESE days it is not sufficient for a neuroscientist to be solely a physiologist, an anatomist, a chemist or a behavioural scientist. Too often, the anatomist fails to realize that not all connections may be functional, the physiologist loses sight of the way an animal behaves and the neurochemist does not put his molecular hypotheses in the context of the functioning brain. For students of neuroscience, this is especially important. Aspects of neurobiology taught in different departments may appear to bear little relation to one another, and much of the excitement of the brain sciences is lost in a string of unrelated facts.

Textbook writers have recently become aware of the problem and we are now witnessing the advent of a new generation of neuroscience texts aimed at overcoming undue segregation of the subject. Neuroanatomy in particular is found by many students to be difficult to assimilate and relate to the living brain; yet without it we are lost, having no framework on which to build. For this reason Jay Angevine and Carl Cotman's Principles of Neuroanatomy is a wonderful book. Aimed to supplement a basic anatomy text such as A. Brodal's Neurological Anatomy (Oxford University Press, 1981) or M.B. Carpenter's Core Text of Neuroanatomy (Williams and Wilkins, 1978) rather than to be used alone, it is a joy to read.

Here is a book that makes neuroanatomy come alive, that brings together all the facts and gives them some meaning. It has a chatty, easy style but the contents are accurate and concise. The sections on

\section{Companion to Kornberg}

W.H. Freeman have issued a 1982 Supplement to Arthui Kornberg's textbook DNA Replication, which was first published in 1980 and reviewed in Nature 289, 718 (1981). The Supplement covers developments in the subject up to January 1982 , and costs $£ 7.50, \$ 10$. thalamus, reticular formation and limbic system are particularly good and there is an up-to-date section on chemical coding. Clinical and psychophysical data are well covered, and there are frequent references, by analogy, to everyday matters.

Another book which can be highly recommended to both student and research worker is the third edition of Basic Neurochemistry. Here the detailed and complex subject of biochemistry of nervous tissue is put in the context of other branches of neuroscience. Coverage ranges from basic neurocellular anatomy and physiology to clinical disorders and behavioural science. There are chapters on neurotransmitters, cell motility, brain energy metabolism and many more topics, all written clearly and concisely with up-to-date references and a good index. Amongst the neurosciences neurochemistry has made some of the greatest advances in the past few decades and that is reflected in the book.

In comparison to these last two books, Stein's An Introduction to Neurophysio$\log y$ seems distinctly old-fashioned. This is particularly disappointing since the author aims to convey "the fascination and excitement" of neurophysiology, which is so lacking in many textbooks. This admirable aim, however, is never realized. The book has a fairly classical description of electrical properties of neurones, sensory and motor systems and goes on to reward, learning and memory. As such it has little to offer over other concise texts such as Fundamentals of Neurophysiology edited by R.F. Schmidt (Springer-Verlag, 1978), and in places is less accurate. There are good chapters, however, those on motor control being the best. The subject is approached from the end-point, a movement, which is then broken down into its constituent parts and the result is successful. But the book as a whole, although perfectly adequate and sound, is never stimulating. The excitement of neurophysiology comes from its attempt to give a functional perspective to anatomy and chemistry and provide a mechanism for behaviour; without this it becomes a series of important but rather dry observations.

Another new book intended as an introductory text is P.J. Mill's Comparative Neurobiology. As such it is inadequate, with very limited treatment of the central nervous system and few references. Where it does score is in its comparative approach. The author describes the anatomy, physiology and pharmacology of nerve and muscle by using constant examples from both vertebrates and invertebrates. This book is not sufficient for undergraduates coming to neuroscience for the first time, but it is full of fascinating facts with particularly good chapters on sensory receptors and on the control of behaviour in different species. It is a book for the true, all-round neuroscientist.

Maria Fitzgerald is a Tutor in Neuroscience and an Associate Research Fellow in the Department of Anatomy, University College London. 